\title{
Cryogenic orthogonal turning of Ti-6Al-4V
}

\section{Analysis of nitrogen supply pressure variation and subcooler usage}

\author{
Benedict Stampfer ${ }^{1} \cdot$ Philipp Golda $^{2} \cdot$ Robert SchießII $\cdot$ Ulrich Maas $^{2} \cdot$ Volker Schulze $^{1}$
}

Received: 23 April 2020 / Accepted: 14 September 2020 / Published online: 28 September 2020

(C) The Author(s) 2020

\begin{abstract}
Cooling of machining operations by liquid nitrogen is a promising approach for reducing cutting temperatures, increasing tool life and improving the workpiece surface integrity. Unfortunately, the cooling fluid tends to evaporate within the supply channel. This induces process variations and hinders the use of nitrogen cooling in commercial applications. In this work, the coolant is applied via the tool's rake face during orthogonal turning of Ti-6Al-4V. The effect of a nitrogen supply pressure adjustment and a subcooler usage - proposed here for the first time for machining — is analyzed in terms of process forces, tool temperatures and wear patterns, taken dry cutting as a reference. Thereby, reliable cooling strategies are identified for cryogenic cutting.
\end{abstract}

Keywords Orthogonal turning $\cdot$ Liquid nitrogen $\cdot$ Adaptive cooling

\section{Introduction}

Cooling strategies of machining processes are economically and ecologically relevant, since high efforts arise for the supply, maintenance and disposal of conventional cutting fluids. Hence, the substitution of these cutting fluids is a promising approach moving towards environmental friendly production. In the machining of low strength materials such as aluminum alloys, dry cutting strategies and minimum quantity lubrication are used [1].

These strategies however are not suited for Ti-6Al-4V, which is a commonly applied titanium alloy and a difficult to machine material [2]. The high strength and low heat conductivity of Ti-6Al-4V lead to high cutting temperatures and diffusion of the chemical reactive titanium into the tool material [3]. This causes adhesion of titanium carbides on the rake of tungsten carbide tools. The adhered material

Benedict Stampfer

benedict.stampfer@kit.edu

1 wbk Institute of Production Science,

Karlsruhe Institute of Technology (KIT),

Kaiserstr. 12, 76131 Karlsruhe, Germany

2 Institute of Technical Thermodynamics (ITT),

Karlsruhe Institute of Technology (KIT),

Kaiserstr. 12, 76131 Karlsruhe, Germany is occasionally torn away by the chip, resulting in crater wear [4]. As those mechanisms are temperature sensitive, a small increase of the cutting parameters may cause rapid tool wear. Arrazola et al. [5] identified a cutting velocity of $90 \mathrm{~m} \mathrm{~min}^{-1}$ as critical value when turning Ti-6Al-4V. Machining with elevated cutting parameters hence demands for effective process cooling. Consequently, machining of Ti-6Al-4V is regarded as a test case for improved cooling concepts in this work.

High pressure cooling is a strategy which is suited for difficult to machine materials, but unfortunately issues associated with the cleaning of parts and energy consumption are critical [2]. An approach which overcomes the needs of maintenance and disposal of lubricants as well as many environmental concerns is the use of liquid nitrogen $\left(\mathrm{LN}_{2}\right)$ as a cooling medium. Nitrogen's low boiling temperature of $-196{ }^{\circ} \mathrm{C}$ at normal pressure and the evaporation enthalpy promise a high cooling potential when applied into the cutting zone.

Nitrogen is among the very few cooling media which is ubiquitary available. Using air liquefaction, $\mathrm{LN}_{2}$ could be generated during overproduction periods of renewable energies, being saved in dewars and consumed when needed. After its application, the gaseous nitrogen discharges into the atmosphere. Hence, $\mathrm{LN}_{2}$ cooling could be used for zero emission machining of Ti-6Al-4V. Considering the goals of developed countries to continuously reduce carbon dioxide 
emissions until the year 2050, such sustainable manufacturing technologies are of utmost industrial significance. In the following section, cryogenic turning of titanium alloys and challenges of $\mathrm{LN}_{2}$ cooling are reviewed.

\section{State of the art}

\subsection{Cryogenic turning of titanium alloys}

The cutting parameters used in the following reviewed literature are given in Table 1 . Hereby, $v_{c}$ denotes the cutting velocity, $f$ the feed rate and $a_{p}$ the cutting thickness. The working pressure is denoted as $p_{w}$ and refers to the static pressure of a nitrogen tank, which is added to the atmosphere pressure.

Hong and Ding [6] compared dry, emulsion and $\mathrm{LN}_{2}$ assisted turning of Ti-6Al-4V. The approaches were investigated experimentally and ordered by means of the occurring tool temperatures (highest to lowest): dry cutting, tool back cooling, emulsion cooling, workpiece precooling, flank cooling, rake cooling and simultaneous rake and flank cooling. In the latter case the nitrogen rate is $6 \mathrm{~g} \mathrm{~s}^{-1}$ for each nozzle, however in workpiece and tool back cooling the rate is $18 \mathrm{~g} \mathrm{~s}^{-1}$. This demonstrates that an efficient medium application leads to a better cooling effect despite a lower nitrogen consumption.

Bermingham et al. [7] investigated the effectiveness of $\mathrm{LN}_{2}$ application during turning of Ti-6Al-4V at a constant cutting velocity of $125 \mathrm{~m} \mathrm{~min}^{-1}$. Cutting with rake and flank cooling was compared with dry cutting. The $\mathrm{LN}_{2}$ application prolonged the tool life between 43 and 58\%. Increasing the feed rate and in terms reducing the cutting thickness to maintain the same material removal rate, drastically reduced the tool life in dry and $\mathrm{LN}_{2}$ cutting.

Klocke et al. [8] investigated the impact of $\mathrm{LN}_{2}$ cooling on the tool life when turning the gamma titanium alloy 452-2XD. Nitrogen was supplied through a rake face nozzle into the cutting zone with a working pressure of 2 bar. Compared with conventional emulsion cooling, minimum quantity lubrication causes a reduction of the tool wear VB of $7 \%$, high pressure cooling causes a reduction of $29 \%$ to $41 \%$, depending on the pressure, and $\mathrm{LN}_{2}$ causes a reduction of $61 \%$. These results confirm the ability of lowering the tool wear by $\mathrm{LN}_{2}$, but the medium consumption of 136.8 $\mathrm{kg} \mathrm{h}^{-1}$ must be considered rather high for commercial application.

Bordin et al. [9] investigated dry and cryogenic turning of Ti-6Al-4V. $\mathrm{LN}_{2}$ was applied into the cutting zone by a rake face and a flank face nozzle. Thereby the nose wear is reduced by $25 \%$ with the parameters cutting velocity $80 \mathrm{~m} \mathrm{~min}^{-1}$, the feed $0.2 \mathrm{~mm}$ and the cutting depth 0.25 $\mathrm{mm}$. After analyzing the tool by optical imaging, scanning electron microscopy and energy dispersive X-ray spectroscopy it was concluded that the main wear mechanism is adhesion for both, dry and cryogenic cutting. $\mathrm{LN}_{2}$ application reduced the tool-workpiece-adhesion by lower cutting temperatures which causes less reactivity between titanium and the tungsten carbide tool. Consequently crater wear is avoided.

Aramcharoen [10] investigated oil and $\mathrm{LN}_{2}$ cooling when turning Ti-6Al-4V. The heat affected tool area and the toolchip-contact-length were significantly reduced by cryogenic cooling, while the chip curl was increased. In cryogenic cutting helical chips were produced, compared with snarled chips with oil cooling. The thermocouple measurements showed a temperature decrease of $200 \mathrm{~K}$ between oil and cryogenic cooling. Unfortunately, this information is difficult to interpret since the thermocouple location and the absolute temperatures are not mentioned.

Trabelsi et al. [11] compared conventional and cryogenic cooling during turning of the titanium alloy Ti17 (Ti$5 \mathrm{Al}-2 \mathrm{Sn}-2 \mathrm{Zr}-4 \mathrm{Mo}-4 \mathrm{Cr}$ ). The cutting parameters of the full factorial experimental design are given in Table 1. Higher cutting parameters generally lead to severe tool degradation, irrespective of the cooling concept used. This especially

Table 1 Literature review of $\mathrm{LN}_{2}$ assisted longitudinal turning of titanium alloys

\begin{tabular}{llllllll}
\hline & $\begin{array}{l}v_{c} \\
\mathrm{~mm} \mathrm{~min}^{-1}\end{array}$ & $\begin{array}{l}f \\
\mathrm{~mm}\end{array}$ & $\begin{array}{l}a_{p} \\
\mathrm{~mm}\end{array}$ & $\begin{array}{l}\text { Rake nozzle } \\
\mathrm{d} \text { in mm }\end{array}$ & $\begin{array}{l}\text { Flank nozzle } \\
\mathrm{d} \text { in mm }\end{array}$ & $\begin{array}{l}p_{w} \\
\mathrm{bar}\end{array}$ \\
$\begin{array}{llllll}\text { Mass flow } \\
\mathrm{g} \mathrm{s}^{-1}\end{array}$ \\
Hong and Ding [6] & 90 & 0.254 & 1.27 & Unknown & Unknown & Unknown & $12^{1}$ \\
Bermingham et al. [7] & 125 & $0.15,0.2,0.36$ & $2.7,2.0,1.1$ & Unknown & 1.77 & 8.3 & Unknown \\
Klocke et al. [8] & 80 & 0.1 & 0.25 & $4.24^{2}$ & Not used & 2 & 38 \\
Bordin et al. [9] & 50,80 & $0.1,0.2$ & 0.25 & 0.9 & 0.9 & 15 & 15 \\
Aramcharoen [10] & 70,100 & 0.25 & 0.5 & Unknown & Unknown & 4 & $5^{1}$ \\
Trabelsi et al. [11] & 50,90 & $0.1,0.3$ & $1.5,3.0$ & $2 * 1.7^{3}$ & Not used & 12 & Unknown \\
\hline
\end{tabular}

\footnotetext{
${ }^{1}$ Recalculated from volumetric stream measurements, assuming $\mathrm{LN}_{2}$ with the density $807 \mathrm{~g} \mathrm{l}^{-1}$

${ }^{2}$ In [8] an elliptic nozzle was used. Recalculated diameter assuming equal cross section areas

${ }^{3}$ Two rake nozzles were used in [11]
} 
accounts for the cutting velocity $90 \mathrm{~m} \mathrm{~min}^{-1}$ which leads to tool life of $1.2 \mathrm{~min}$ or less. When comparing the cooling concepts at the cutting velocity $50 \mathrm{~m} \mathrm{~min}^{-1}$ and the feed 0.1 $\mathrm{mm}$, the tool life under cryogenic cooling increases from 23 to $35 \mathrm{~min}$ for the cutting depth $1.5 \mathrm{~mm}$ and from 15 to $29 \mathrm{~min}$ for the cutting depth $3 \mathrm{~mm}$. The effect of the cutting parameters on the tool wear was standardized and analyzed. Increasing the cutting depth has the lowest effect on the tool wear, while increasing the feed has the highest effect. Thereby, the findings of Bermingham et al. [7] were confirmed.

Generally tool wear could be reduced by $\mathrm{LN}_{2}$ cooling, but the application system, the nozzles and the working pressures considerably differ (see Table 1). This leads to mass flow rates between 5 and $38 \mathrm{~g} \mathrm{~s}^{-1}$. Cutting temperatures were only measured in rare cases. Yet, such knowledge would allow to adjust the nitrogen consumption, depending on the aspired process temperatures. Achieving such knowledge is pursued in the present work.

\subsection{Challenges of $\mathrm{LN}_{2}$ cooling}

In cryogenic machining, $\mathrm{LN}_{2}$ inevitably tends to evaporate within the supply channels. As a countermeasure insulated pipes [6, 8, 9], insulated tools [10] or a phase separator [11] were used. The effectiveness of those actions has not been analyzed in the referring articles.

Dix et al. [12] explicitly addressed the problem of evaporation in the case of $\mathrm{LN}_{2}$ cooled drilling. The internal channels of the investigated twist drills had a diameter of $1.2 \mathrm{~mm}$ each, which were supplied with a working pressure of 1.3 bar. At the tool tip, the boiling temperature of nitrogen was reached only after a precooling duration of $9 \mathrm{~min}$. This was followed by periodic temperature increases of more than $80 \mathrm{~K}$, which were explained by nitrogen evaporation within the tool channels.

Busch et al. [2] compared the concepts high pressure cooling, nitrogen cooling, carbon dioxide cooling and aerosol dry lubrication combined with carbon dioxide cooling for difficult to machine materials. As major obstacles for the application of nitrogen cooling, the initial cooling duration and the phase variations were named.

Pusavec et al. [13] confirmed the phenomenon of nitrogen evaporation in the supply system by the integration of an optical phase sensor in an $\mathrm{LN}_{2}$ delivery nozzle. An additional thermocouple sensor measured the temperature of the nitrogen stream. The measured phase variations at the beginning of the cooling process correlated with temperature peaks. Even after a stationary temperature state was reached, occasional phase variations were indicated. Hence, those variations are principle problems of nitrogen cooling, which is not completely avoided by initial precooling. $\mathrm{LN}_{2}$ evaporation in the supply channel can impair the cooling effect, because the heat transfer coefficient between gas and a solid body in forced convection is usually less than $0.1 \mathrm{~kW} \mathrm{~K}^{-1} \mathrm{~m}^{-2}$, whereas it reaches $1 \mathrm{~kW} \mathrm{~K}^{-1} \mathrm{~m}^{-2}$ for $\mathrm{LN}_{2}$ film boiling and values higher than $10 \mathrm{~kW} \mathrm{~K}^{-1} \mathrm{~m}^{-2}$ for an impinging $\mathrm{LN}_{2}$ jet [14]. Consequently the nitrogen evaporation tendency is a main issue for a reliable process cooling.

Subcooling is a known principle when $\mathrm{LN}_{2}$ is used to cool silicone chips in microelectronics. Vader et al. [15] investigated the heat flux of a chip in a nitrogen bath with different subcooling levels. By subcooling $\mathrm{LN}_{2}$ by $3.6 \mathrm{~K}$, the heat flux increased from less than 15 to $30 \mathrm{~W} \mathrm{~cm}^{-2}$. Subcooling can avoid the evaporation in the supply system by decreasing of the nitrogen temperature below the boiling state. Subcooling should also reduce nitrogen evaporation in supply lines, but to the knowledge of the authors it has not been investigated in the application of cryogenic cutting.

Lequien et al. [16] investigated the cooling effect of an $\mathrm{LN}_{2}$ jet on a Ti-6Al-4V workpiece. To measure its temperature, thermocouples are positioned beneath the $\mathrm{LN}_{2}$ impinged surface. Experimental input parameters for the cooling tests are the nozzle diameter $(1.5$ and $3 \mathrm{~mm})$, working pressure ( 2 and 6 bar), nozzle angle ( 15 and $45^{\circ}$ inclination to the workpiece normal) and the nozzle-workpiece distance (15 and $100 \mathrm{~mm})$. In those tests, increasing the nozzle diameter is the dominant factor to increasing the cooling rate, followed by the increase of the working pressure. In CFD simulations, heat transfer coefficients between 8825 $\mathrm{W} \mathrm{K} \mathrm{K}^{-1} \mathrm{~m}^{-2}$ and $15630 \mathrm{~W} \mathrm{~K}^{-1} \mathrm{~m}^{-2}$ were calculated, depending on the experimental input parameters. The nitrogen phase ratio was a free parameter of the simulations, which was chosen iteratively. With a proportion of $90 \mathrm{wt} \%$ liquid and $10 \mathrm{wt} \%$ gas phase a good matching was achieved between experimental and simulated cooling rates.

In subsequent work, Lequien et al. [17] investigated interrupted turning of Ti-6Al-4V with the concepts $\mathrm{LN}_{2}$ cooling, flood cooling and dry cutting. In cryogenic cutting a working pressure of 2 bar was used to supply $\mathrm{LN}_{2}$ to rake and flank face. The flank nozzle with the diameter 1.2 $\mathrm{mm}$ remained fix, while rake nozzles with the alternative diameters 1,2 or $3 \mathrm{~mm}$ were tested. This resulted in $\mathrm{LN}_{2}$ total mass flow rates of 16.1, 25.6 and $33.6 \mathrm{~g} \mathrm{~s}^{-1}$. In order to measure the machining temperatures, a thermocouple sensor was positioned $1 \mathrm{~mm}$ beneath the tool surface in a distance of $1 \mathrm{~mm}$ to the nose of the cutting tool. For a selected parameter set, dry cutting lead to a tool temperature of approximately $200{ }^{\circ} \mathrm{C}$, flood cooling to $100{ }^{\circ} \mathrm{C}, \mathrm{LN}_{2}$ cooling with $1 \mathrm{~mm}$ nozzle diameter to $0{ }^{\circ} \mathrm{C}$ and $\mathrm{LN}_{2}$ cooling with $3 \mathrm{~mm}$ nozzle diameter to $-50^{\circ} \mathrm{C}$. It is worth noticing that the passive force is correlated to the cutting concept, i.e. is raises with decreasing cutting temperature.

A shortcoming of nitrogen cooling is that the medium is lost, once it was used. Hence, it would be beneficial to 
adapt the nitrogen consumption and the cooling effect to the process. The reviewed literature shows that an adjustment by different nozzle diameters as in [17] is possible, however it is not flexible enough for a widespread application. Therefore in this work, it is pursued to adjust the mass flow rate and the process cooling by the working pressure in the nitrogen tank. Furthermore, it is elucidated how the usage of a subcooler affects the process cooling, regarding the issue of the varying phase ratio in the applied nitrogen stream. As this contributes to the generation of fundamental knowledge, the machining process orthogonal turning is investigated.

\section{Experimental setup}

\subsection{Cutting tests}

Dry and cryogenic orthogonal turning of Ti-6Al-4V is presented in this paper. In order to measure the process forces and the tool temperatures, a vertical numerical control turning machine of the type Index V100 was equipped with a dynamometer of the type Kistler 9257B, three type $\mathrm{N}$ thermocouples and a data acquisition module of the type Omega OMB-DAQ-2408. The experimental setup is depicted in Figs. 1 and 2.

In Tables 2 and 3, the machining and the cooling parameters of the fully factorial design of experiments are summarized. Hereby, $h$ is the uncut chip thickness, $w$ the cutting width, $l_{c}$ the length of cut of ten fillets, $\alpha$ the flank angle and $\gamma$ the rake angle. During each experiment ten fillets of the slotted workpiece were consecutively turned, resulting in $24 \mathrm{~m}$ length of cut. For each level of machining parameters at least three experiments were carried out. A new cutting edge was used for each three experiments. The maximum material removal rate of the literature presented in Table 1 is $50 \mathrm{~cm}^{3} \mathrm{~min}^{-1}$ [7], while it is $80 \mathrm{~cm}^{3} \mathrm{~min}^{-1}$ in this work. It can be assumed that effects and benefits of the

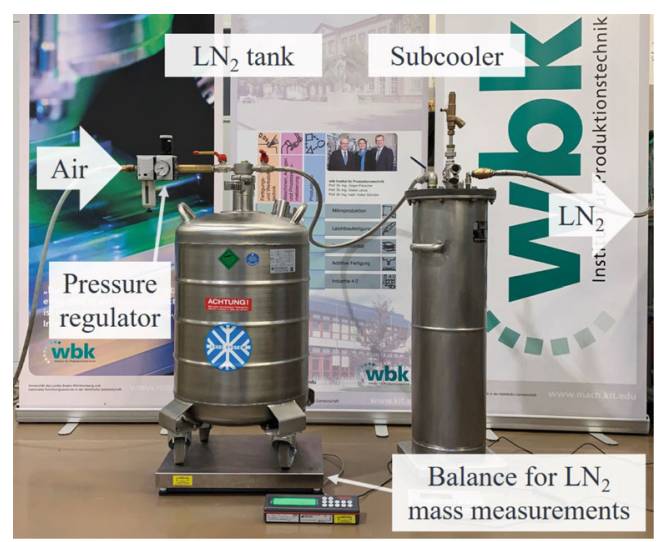

Fig. 1 Setup of the nitrogen supply with subcooler

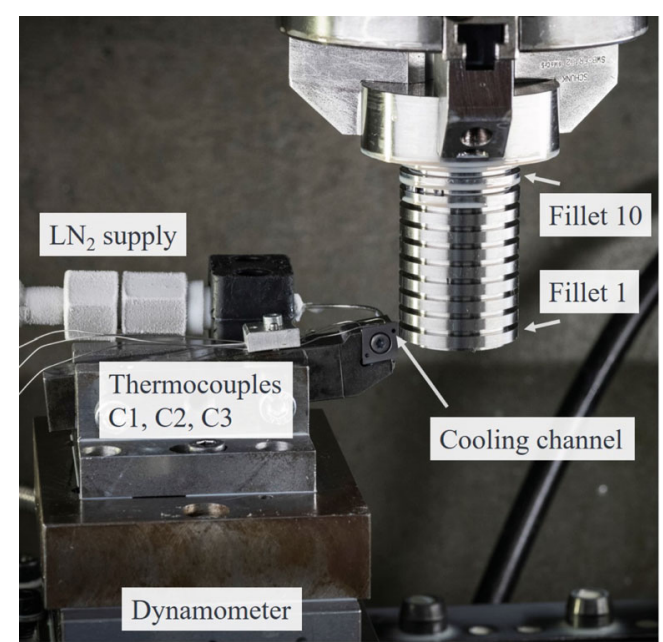

Fig. 2 Setup of the orthogonal cryogenic turning tests

pressure variation or the subcooler, which are presented in the following, are also relevant for less challenging cutting conditions.

A tool holder of the type SCLCR2020K12 was used, equipped with uncoated tungsten carbide inserts of the type CCMW120404, according to DIN ISO 1832. The tool system has been supplied by Walter AG and was customer modified by electrical discharge machining. Geometric features of the cutting tool and the thermocouple positions $\mathrm{C} 1, \mathrm{C} 2$ and $\mathrm{C} 3$ are given in Fig. 3 and Table 4. The thermocouple sensor $\mathrm{C} 1$ was placed in the insert, flushing the tip with the flank face. A direct contact of the thermocouples with $\mathrm{LN}_{2}$ is avoided.

The cooling medium is applied to the cutting process via a tool internal channel by an outlet on the rake face with the diameter $1.5 \mathrm{~mm}$. Rake face application was chosen because it promises the strongest reduction in tool temperatures, compared with exclusively cooling the flank or precooling the workpiece [6]. Additionally cooling the flank face was neglected, because the amount of nitrogen couldn't be separated when two outlets were used. Furthermore it would be impossible to separate the tool cooling effects of rake cooling and flank cooling. This would have made the presented results less understandable and reproducible. A minimum channel-to-edge-distance of $0.8 \mathrm{~mm}$ was chosen to achieve a sufficient tool stability while injecting $\mathrm{LN}_{2}$ close to the cutting zone. Given the cutting width and the circular channel geometry, the distance increases along the cutting edge to values of $1.5 \mathrm{~mm}$ and above. Previously

Table 2 Machining parameters

\begin{tabular}{llllll}
\hline$v_{c}$ & $h$ & $w$ & $l_{c}$ & $\alpha$ & $\gamma$ \\
$\mathrm{m} \mathrm{min}^{-1}$ & $\mathrm{~mm}$ & $\mathrm{~mm}$ & $\mathrm{~m}$ & $\circ$ & $\begin{array}{l}\text { o } \\
100\end{array}$ \\
\hline 0.2 & 4 & 24 & 7 & 0 \\
\hline
\end{tabular}


Table 3 Cooling parameters of the cutting tests (full factorial variation)

\begin{tabular}{ll}
\hline Factor & Levels \\
\hline cooling concept & dry, $\mathrm{LN}_{2}$ no subcooler, $\mathrm{LN}_{2}$ with subcooler \\
$p_{w}$ in bar & $4,5,6,7$ (only for $\mathrm{LN}_{2}$ cooling) \\
\hline
\end{tabular}

published numerical simulations suggest that this should not impair the cooling effect [18].

A crucial factor of undesired environmental heat transfer in a cooling system are lines, which carry the medium. To connect the cryogenic equipment, corrugated stainless steel hoses with a length of $1.5 \mathrm{~m}$ and an inner diameter of 10 $\mathrm{mm}$ were used. In the case of cutting without subcooler, tank and tool were connected by one hose; otherwise, two hoses were used. To transfer $\mathrm{LN}_{2}$ into the cutting tool, a stainless steel pipe with a length of $90 \mathrm{~mm}$ and an inner diameter of $1.4 \mathrm{~mm}$ was used. Given that the heat transfer of a tube is proportional to its surface area, the corrugated hoses are decisive for the evaporation tendency of the $\mathrm{LN}_{2}$. Thus they were customized by the insertion of PTFE-tubes with an inner diameter of $8 \mathrm{~mm}$. The subcooler considerably elongates the cooling medium transfer by a pipe system length of approximately $20 \mathrm{~m}$. However, these pipes are cooled down, as they carry pressurized nitrogen and are plunged into $\mathrm{LN}_{2}$ with ambient pressure.

\subsection{Cooling parameter variation}

A schematic illustration of the nitrogen supply system is given in Fig. 4 based on an exemplary working pressure of $5 \mathrm{bar}$ and hence of a total tank pressure of $p_{1}=6 \mathrm{bar}$.

The nitrogen stored in the cryogenic tank is initially in a boiling state. Since the heat flux $\dot{q}_{\mathrm{amb}}$ from the environment to the supply system cannot be completely prevented, evaporation of $\mathrm{LN}_{2}$ occurs in the supply pipes

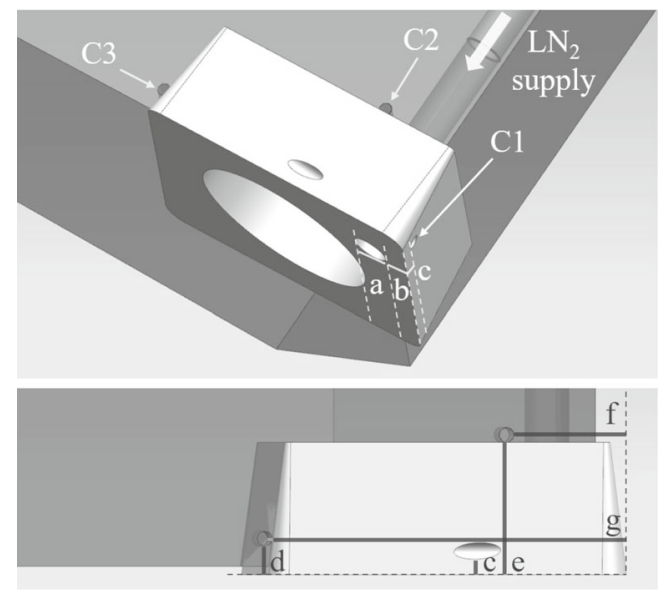

Fig. 3 Tool configuration for cryogenic turning
Table 4 Geometric parameters of the tool system

\begin{tabular}{lllllll}
\hline $\begin{array}{l}\mathrm{a} \\
\mathrm{mm}\end{array}$ & $\begin{array}{l}\mathrm{b} \\
\mathrm{mm}\end{array}$ & $\begin{array}{l}\mathrm{c} \\
\mathrm{mm}\end{array}$ & $\begin{array}{l}\mathrm{d} \\
\mathrm{mm}\end{array}$ & $\begin{array}{l}\mathrm{e} \\
\mathrm{mm}\end{array}$ & $\begin{array}{l}\mathrm{f} \\
\mathrm{mm}\end{array}$ & $\begin{array}{l}\mathrm{g} \\
\mathrm{mm}\end{array}$ \\
\hline 1.5 & 0.8 & 0.5 & 1.5 & 4.8 & 3.9 & 12.7 \\
\hline
\end{tabular}

without subcooler (red path) [13]. Thus, the nitrogen vapor content tends to increase with the distance of travel owing to imperfect heat insulation. This leads to a multi-phase mixture at the nozzle outlet. To avoid this, subcooling of the nitrogen in the pipe system was performed, which is illustrated along the blue path. The supplied nitrogen is subjected to the working pressure and thus has an elevated boiling temperature [19]. Consequently subcooling can be performed using a subcooler containing liquid nitrogen at ambient pressure. Noticing that ambient pressure prevails in the subcooler and thus $T_{\text {boil,sub }}$ is lower than $T_{1}$ in the cryogenic tank, heat flows ( $\left.\dot{q}_{\text {sub }}\right)$ from the pressurized nitrogen to the not pressurized nitrogen in the subcooler. The pressure of the subcooler is maintained by a relief valve. A copper coil is used for the heat exchange in the subcooler because of its high thermal conductivity.

Measurements of the nitrogen mass flow rate in free jet conditions are performed with and without the subcooler for

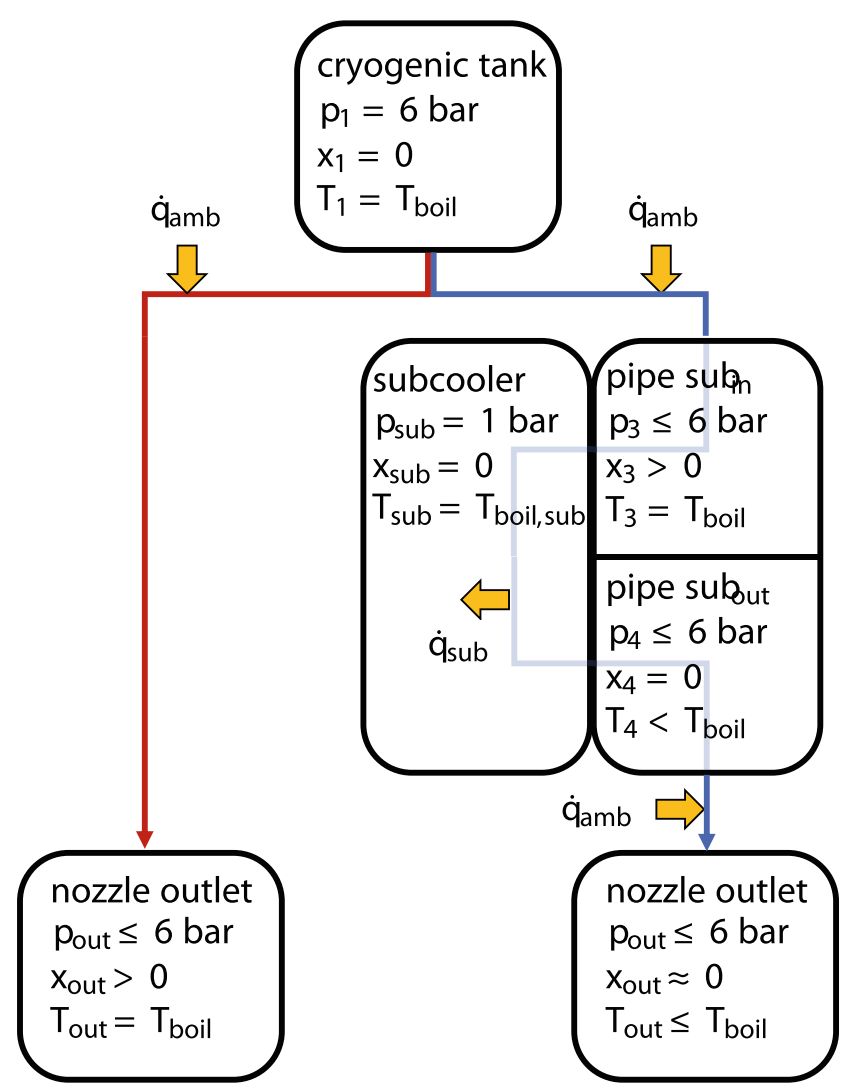

Fig. 4 Comparison of standard nitrogen supply (red line) and the subcooler case (blue line). Example values for the working pressure $p_{w}=5$ bar 


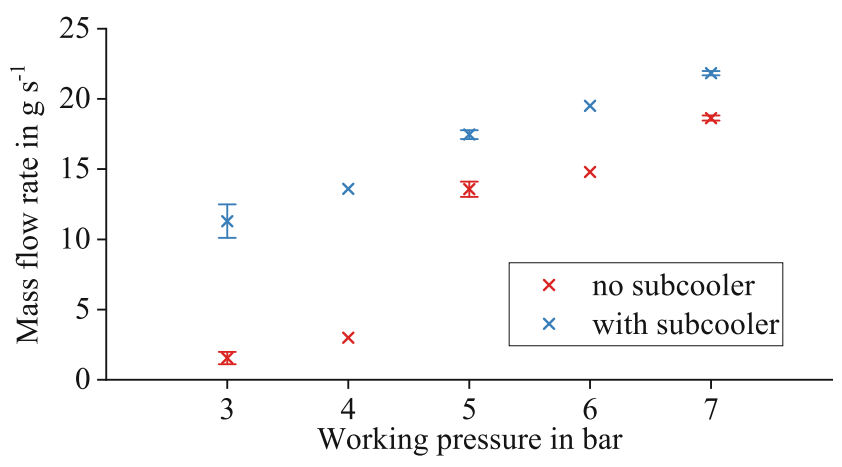

Fig. 5 Dependence of the $\mathrm{LN}_{2}$ mass flow on the working pressure and the subcooler usage for free jet conditions

different working pressures, in order to classify the results of the machining tests in Section 4. These measurements are necessary, since a indication of the working pressure is by no means sufficient because the windage of the system depends on the supply lines [20]. Figure 5 illustrates the measured mass flow rates for different working pressures. As the working pressures 3,5 and 7 bar represent the boundaries and mean value of the parameter space, the measurements were repeated and standard deviations are given.

A significant increase in the mass flow rates can be observed by the application of the subcooler for the entire pressure range. This reveals that nitrogen vapor certainly exists in the supply when the subcooler is not used. Without subcooler, a stationary thermal state cannot be reached for the working pressures 3 and 4 bar. Large amounts of evaporating nitrogen in the supply lines lead to very low mass flow rates. Here the subcooler avoids nitrogen evaporation and drastically increases the mass flow rates. For the given experimental setup, a working pressure higher or equal to 5 bar allows to reach a stationary thermal state without subcooler. But even for the case of 7 bar working pressure, a roughly $20 \%$ gain in the mass flow rate can

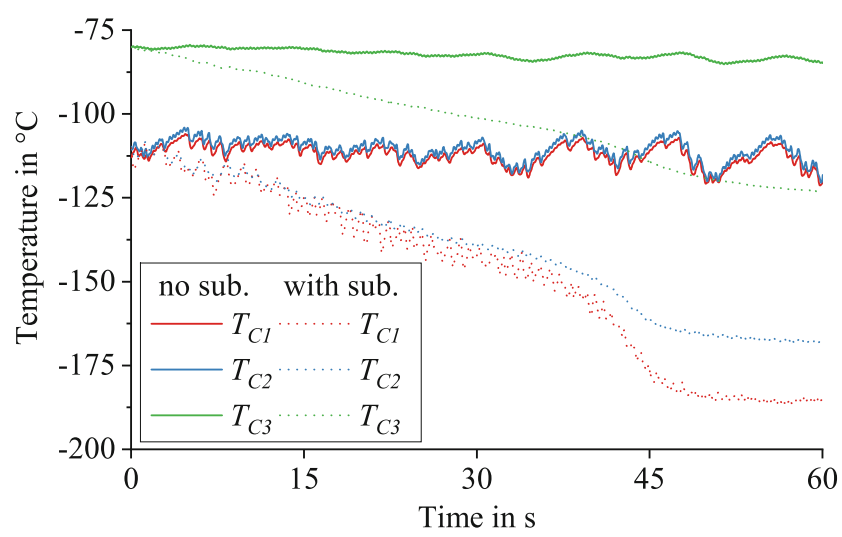

Fig. 6 Decreasing temperatures at the thermocouples C1, C2 and C3. Solid lines indicate no subcooler usage. Dashed lines indicate subcooler usage

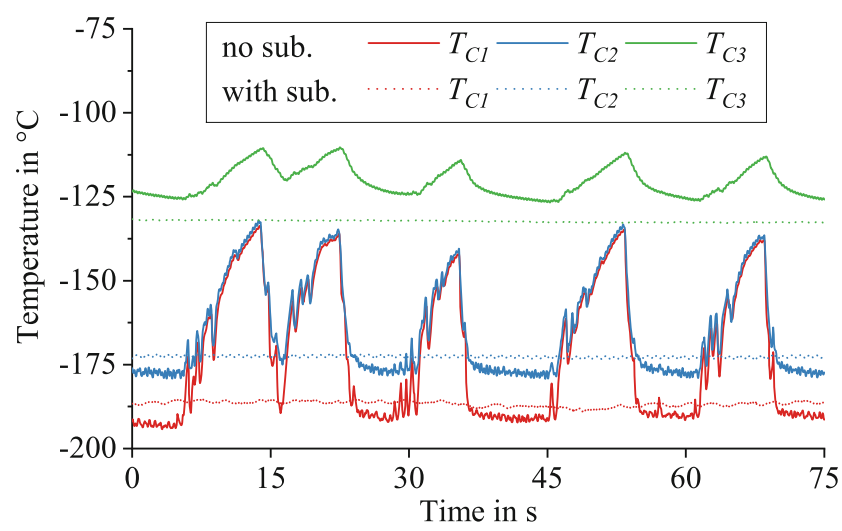

Fig. 7 Resulting temperatures at the thermocouples C1, C2 and C3. Solid lines indicate no subcooler usage with a precooling time of 30 min. Dashed lines indicate subcooler usage with a precooling time of less than $5 \mathrm{~min}$

be achieved with the subcooler, which is encouraging in terms of an enhanced cooling performance. Here, it must be noticed that the mass flow rates during the cutting process are significantly reduced compared with the free jet conditions, because the windage of the system increases due to the chip formation in front of the nitrogen outlet. For all setup conditions, the mass flow rates approximately change to $30 \%$ compared with the free jet conditions.

A fundamental positive effect of higher mass flow rates is the drastic reduction of the precooling duration, which is needed to decrease the tool temperature from ambient conditions. Figure 6 shows the temperature development at the three thermocouples $\mathrm{C} 1, \mathrm{C} 2$ and $\mathrm{C} 3$ mentioned in Fig. 3. As cooling conditions, the working pressure 3 bar was applied with and without subcooler usage. The temperature runs are compared after $\mathrm{C} 3$ had reached $-80^{\circ} \mathrm{C}$. Without subcooler usage (solid lines), the temperatures barely drop within the considered $70 \mathrm{~s}$. In contrast, the temperatures massively decrease for the subcooling case (dashed lines). The sharp temperature drop at $t=40 \mathrm{~s}$ is due to the insulating effect of ice layers that are formed on the pipes respectively at the indexable insert by the deposition of water vapor in the surrounding at low temperatures.

Prior to the turning process, a statistically steady state should be reached, i.e. the mean temperatures at the thermocouples stay unchanged. In Fig. 7 the referring temperatures are shown for the subcooling (dashed lines) and the non-subcooling (solid lines) case. However, for the non-subcooling case no stationary case was reached despite a precooling time of $30 \mathrm{~min}$. Temperature jumps of up to $60 \mathrm{~K}$ were observed in the thermocouples close to the nitrogen outlet. The nitrogen evaporation in the supply line occasionally results in an outlet of almost pure gas phase yielding to higher temperatures. Those temperature variations are undesired, because a constant cooling performance is not ensured during machining. 
At low working pressures without subcooler usage, a bad reproducibility is expected for the temperatures in a machining process. In contrast, the represented temperatures stay constant when using the subcooler. Furthermore, the illustrated curves were reached in less than $5 \mathrm{~min}$. This is a decisive advantage to increase the productivity, regarding the industrial application of cryogenic cutting. However, at first glance it is noticeable that the lowest temperatures are achieved by the fluctuating supply without subcooler usage. A possible explanation is that during $30 \mathrm{~min}$ insulating ice layer has also formed on the indexable insert. Similar precooling durations with subcooler would also lead to a further temperature decrease by a few degrees. Due to the the difficulties of reaching a stationary thermal state without subcooler at low working pressures, it was decided to analyze cutting experiments with $p_{w}=4,5,6$ and 7 bar.

\section{Results}

\subsection{Force measurements}

In Fig. 8 the force measurement of a cryogenic turning experiment $\left(p_{w}=5\right.$ bar, no subcooler $)$ is depicted as a typical example. Herein, $F_{c}$ and $F_{p}$ denote the cutting and the passive force. Higher forces at the beginning of certain cuts are running-in-effects of the engaging tool. The peaks which occur at the end of the cuts 2 to 10 are a consequence of the experimental setup and result from the tool sliding on the previously cut fillet. To overcome those effects, the force measurement of one tool engagement was averaged over a time span of $0.9 \mathrm{~s}$, which is indicated by the green background. The forces were averaged to a joint mean value for each parameter set and a standard deviation, which reflects the variation of the mean values between the experiments.

In Fig. 9 the averaged process forces are summarized. In dry turning, the mean cutting and passive forces are

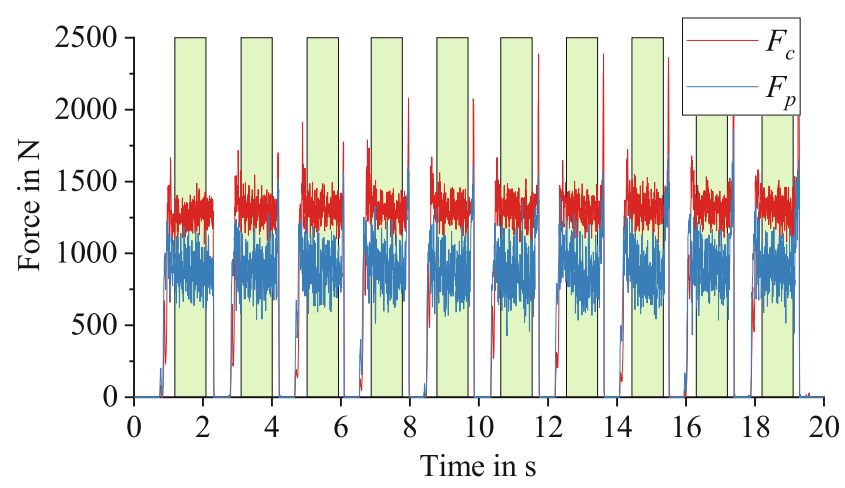

Fig. 8 Process forces and averaging periods (green background) of a single experiment approximately $5 \%$ and $14 \%$ higher than in cryogenic turning. Comparing the values in cryogenic turning, cooling with 4 bar without subcooler leads to the highest passive forces. Under this cooling condition, a stationary temperature state was hardly reached. The insufficient cooling causes high cutting temperatures and an increased tool wear, which is investigated in the following sections. Consequently this cooling condition leads to passive forces in between cryogenic and dry turning. It is interesting to notice that the cooling condition 7 bar with subcooler leads to relatively high passive process forces. This may contribute to Pusavec et al. [21], who state that too low temperatures in cryogenic machining of Ti-6Al-4V impair the cutting process and decrease the tool life time. This phenomenon will be subjected to further investigations.

It must be noticed that the passive forces in dry turning are comparable to cryogenic turning during the first experiment. Yet, they strongly increase during the subsequent dry cutting tests, which is reflected by the high standard deviation and is linked to proceeding tool wear. The main wear mechanism in the machining of Ti$6 \mathrm{Al}-4 \mathrm{~V}$ with carbide tools is tool-workpiece-adhesion [9]. In cryogenic machining a lower temperature can decrease forces by reducing adhesion-diffusion mechanisms and diminishing the adhesion area itself. Furthermore the direct lubrication effect of an $\mathrm{LN}_{2}$ film, which was identified in pin-on-disc tests in [22] could decrease tangential stresses in those parts of rake face, where coulomb friction occurs. In Section 4.3 the cutting edges used in this paper are analyzed with respect to the mentioned mechanisms.

Supplementary tests with the depth of cut $0.1 \mathrm{~mm}$ were conducted, comparing dry with cryogenic turning with 5 bar working pressure and subcooler usage. Here the mean cutting and passive forces of cryogenic turning were $13 \%$ and $11 \%$ lower than for dry turning. An explanation for the cutting force reduction being stronger than with the depth of cut $0.2 \mathrm{~mm}$ is that lubrication becomes more important for

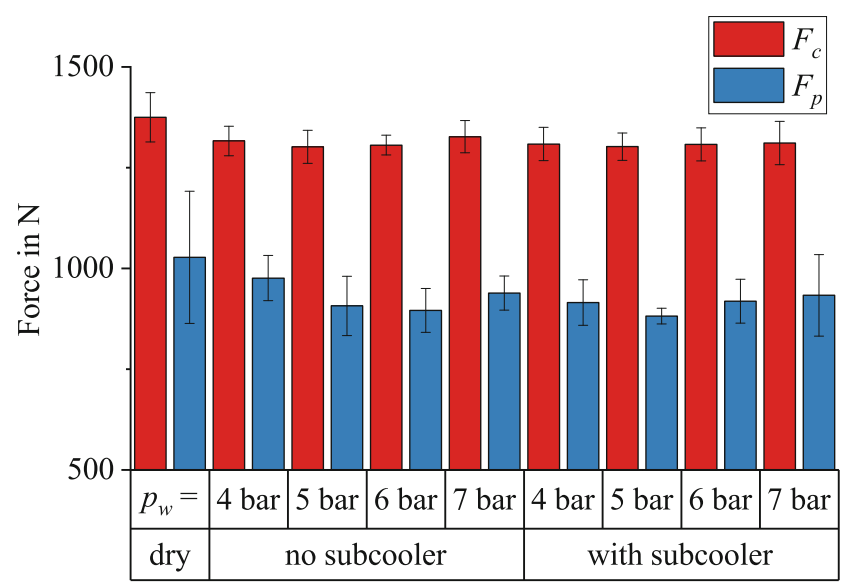

Fig. 9 Averaged process forces 
smaller depths of cut. The passive force reduction is lower than with the depth of cut $0.2 \mathrm{~mm}$ due to the lower impact of the tool wear when dry turning with $0.1 \mathrm{~mm}$. Generally, a process force reduction between 5 and $15 \%$ could be achieved by cryogenic cooling. Irrespective of the cooling strategy, a stronger decrease of the cutting forces is hardly expected with unworn tools, due to the adhesion between Ti$6 \mathrm{Al}-4 \mathrm{~V}$ and carbide tools, which blocks lubricating fluids.

\subsection{Temperature measurements}

In Figs. 10, 11 and 12 typical temperature measurements of dry and cryogenic cutting experiments are depicted. The signals of the thermocouple sensors differ systematically. Sensor C1 generally reaches the highest temperatures. The temperature sharply increases at the beginning of each cut and decreases after each cut. At sensor $\mathrm{C} 2$, the basic temperature increase is superposed by small peaks and at sensor $\mathrm{C} 3$ an almost monotone temperature increase occurs. This behavior is consistent with the sensor positions. A larger distance to the cutting zone decreases and dampens the temperature profile during the experiment.

In dry cutting, the temperature of sensor $\mathrm{C} 1$ increases steadily with each cut, reaching a plateau around $560{ }^{\circ} \mathrm{C}$. In cryogenic cutting without subcooler, the maximum temperatures at sensor $\mathrm{C} 1$ during the cuts vary significantly, reaching values between 300 and $400{ }^{\circ} \mathrm{C}$. A sharp drop occurs between the third and fourth cut. This behavior is explained by a phase variation of the supplied nitrogen. In the experiment with subcooler usage, the maximum temperatures of sensor $\mathrm{C} 1$ are only varying to a small degree. Within the last three cuts a plateau around $290{ }^{\circ} \mathrm{C}$ is reached. After the last cut, the sensor temperatures decrease faster than in the experiment without subcooler usage. The results presented show that the subcooler clearly stabilizes the process cooling. Without using a subcooler, the higher fraction of gaseous nitrogen in the medium stream causes a sporadic, unpredictable increase of the measured temperature. These findings are in accordance with [12] and [13].

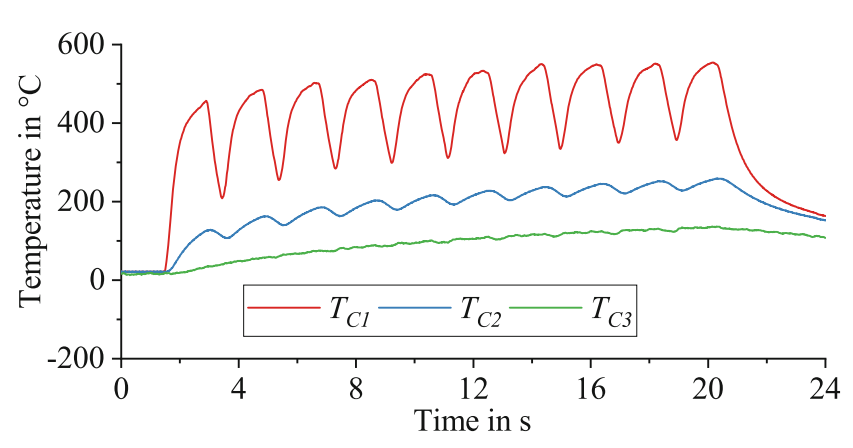

Fig. 10 Temperature measurement of dry cutting

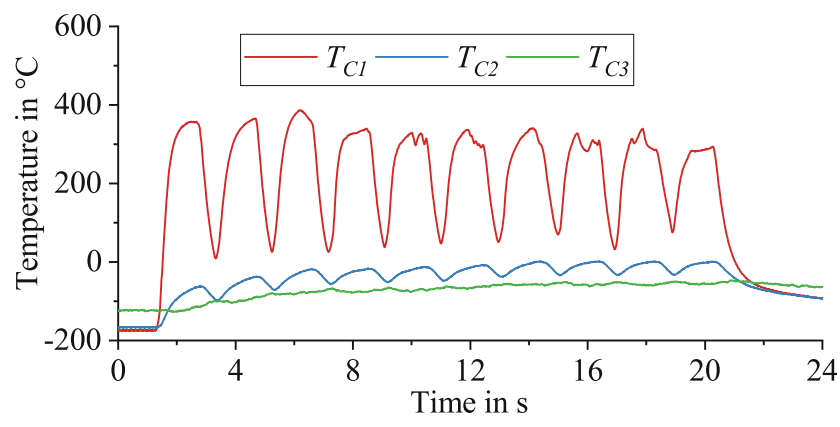

Fig. 11 Temperature measurement of cryogenic cutting with $p_{w}=7$ bar and no subcooler usage

Since the sensor $\mathrm{C} 1$ has the smallest distance to the cutting zone, its values are the most relevant ones for the process and the tool wear. For a general comparison, the maximum temperature of sensor $\mathrm{C} 1$ was evaluated for each cutting experiment. The temperatures were averaged to a joint mean value for each parameter set and a standard deviation, which reflects the variation between the experiments. As depicted in Fig. 13, the temperatures in dry cutting reach $550{ }^{\circ} \mathrm{C}$, while in cryogenic cutting the values range from 300 to $480^{\circ} \mathrm{C}$.

Cryogenic cutting without subcooler and a working pressure of 4 bar results in temperatures of more than $450{ }^{\circ} \mathrm{C}$. Those high maximum temperatures correlate with the high passive forces and are attributed to the strong phase variations of the supplied nitrogen. As stated in Section 3.2, the working pressures 3 and 4 bar without subcooler lead to mass flow rates of less than $5 \mathrm{~g} \mathrm{~s}^{-1}$ and a stationary temperature state cannot be reached. Due to insufficient process cooling, the tool wear is also expected to lie in between dry turning and the remaining cryogenic turning tests. For 4 bar working pressure, subcooler usage decreases the maximum temperatures to approximately $360{ }^{\circ} \mathrm{C}$. This effect is explained by the strong reduction of phase variations in the nitrogen stream, which was also recognized in the precooling phase. Starting from room temperature, a stationary temperature state was reached within approximately $5 \mathrm{~min}$ by a mass flow rate according increasing to more than $10 \mathrm{~g} \mathrm{~s}^{-1}$.

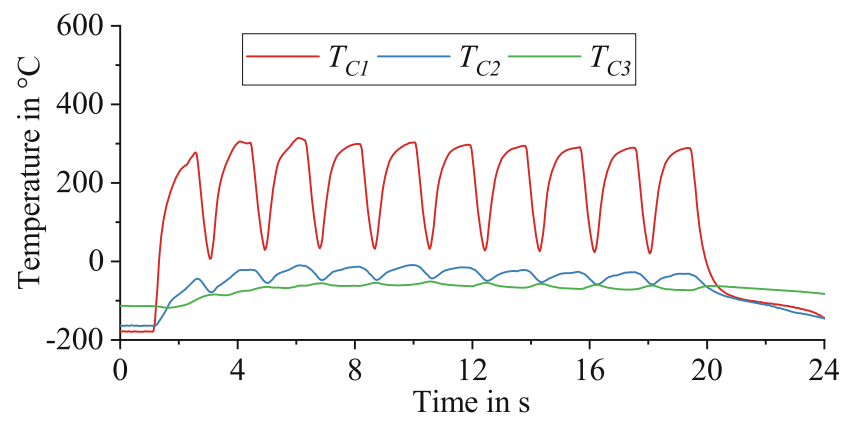

Fig. 12 Temperature measurement of cryogenic cutting with $p_{w}=7$ bar and subcooler usage 


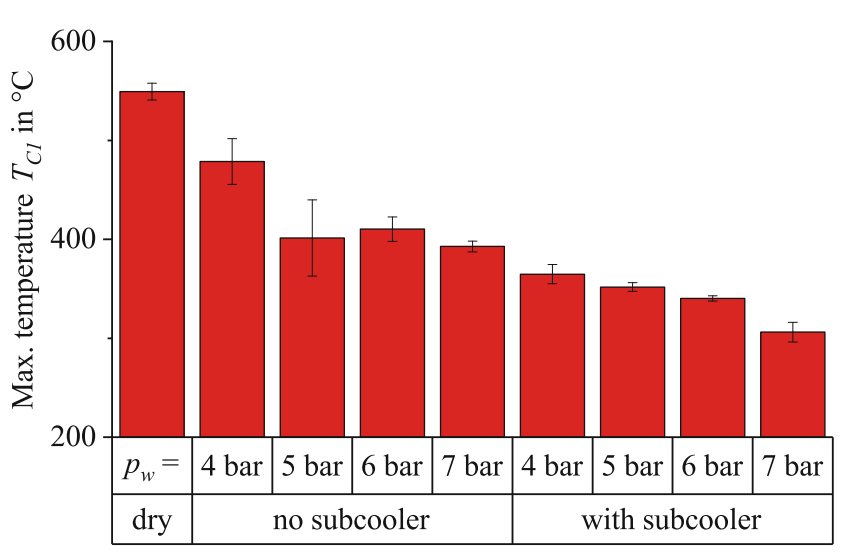

Fig. 13 Maximum temperatures of thermocouple $\mathrm{C} 1$

In cryogenic cutting without subcooler and 5 bar working pressure, the mean maximum temperature is approximately $400{ }^{\circ} \mathrm{C}$ while the standard deviations are very high, despite six repetitions, which were conducted in this case. This reflects an unsteady cooling process between the experimental repetitions. For the given experimental setup without subcooler, working pressures up to 5 bar lead to a bad reproducibility, because the nitrogen flow is influenced by the insulating state of the supply lines. Using the subcooler at this working pressure decreases the mean maximum temperature to approximately $350{ }^{\circ} \mathrm{C}$ and leads to much lower deviations. Again this contributes to the lower nitrogen stream variations.

Cryogenic cutting without subcooler at the working pressures 6 and 7 bar leads to mean maximum temperatures between 390 and $410{ }^{\circ} \mathrm{C}$. Especially at 7 bar working pressure the temperature deviation among experimental repetitions is low. Yet, the temperature variation during one experiment is high, which was shown in Fig. 11 and is caused by nitrogen evaporation in the supply lines. A decreasing temperature trend is seen when translating from the working pressure 6 to 7 bar, which may be explained by the increased $\mathrm{LN}_{2}$ mass flow rate. However, taking into account the temperature deviations between and within the experiments, a temperature control by the working pressure without subcooler is difficult to imagine. That applies even more, when the tool temperatures of the working pressure 5 bar are considered, which can be similar to 6 or 7 bar. It is concluded that a certain working pressure level is necessary for an effective cryogenic cooling without subcooler. Otherwise a stationary thermal state is not reached, which leads to an insufficent process cooling as presented with the working pressure 4 bar. A further increase of the working pressure did not lead to a reliable reduction of the mean maximum temperatures.

When using a subcooler, the evolution of the cutting temperatures is easily comprehensible. By rising the working pressure, the nitrogen mass flow rate increases. The

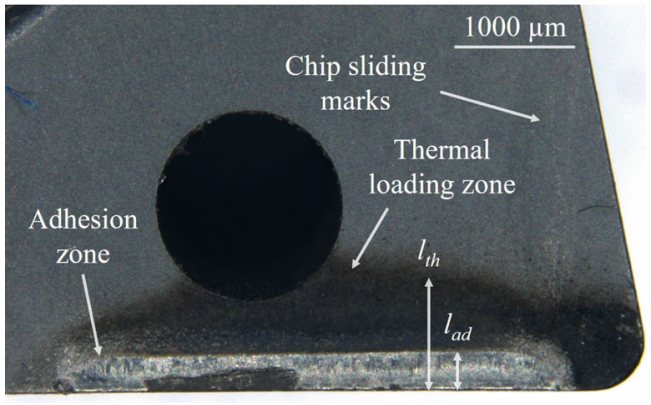

Fig. 14 Tool rake face after $72 \mathrm{~m}$ of cryogenic cutting with $p_{w}=7 \mathrm{bar}$ and no subcooler usage

pressurized nitrogen in the subcooler is chilled to values below the boiling temperature, which avoids evaporation in the subsequent line to the cutting tool. The higher rate and velocity of the nitrogen stream improves its cooling effect. This enables a predictable adjustment of the cutting temperatures by means of the working pressure. Subcooler usage also leads to a faster initial cooling of the system. The subcooler has a very strong effect on the process temperatures when a thermal stationary state is not reached without subcooler. Furthermore the subcooler is effective for higher working pressures as well, because it relies on boiling temperature differences due to the nitrogen pressure. Consequently cryogenic machining with subcooler and 7 bar working pressure led to the lowest maximum temperatures of approximately $310^{\circ} \mathrm{C}$.

\subsection{Tool wear analysis}

A microscopic tool analysis was conducted after the cutting experiments. The inserts depicted in Figs. 14 and 15 are typical examples of cryogenic cutting. For reasons of comparison, the insert of the dry cutting experiments is depicted in Fig. 16. On the tool rake faces three sectors can be distinguished:

- Tool-workpiece-adhesion is marked by a light metallic strap at the cutting edge.

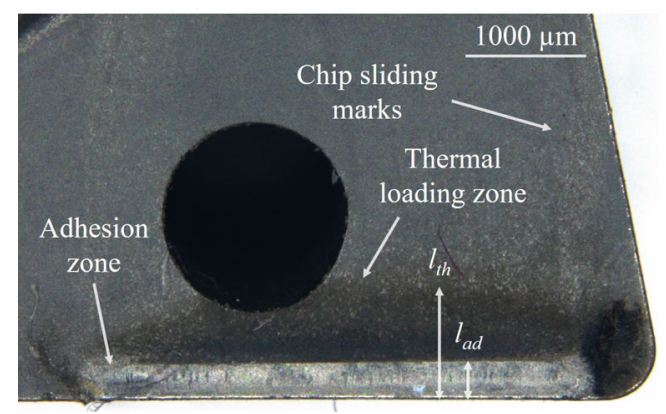

Fig. 15 Tool rake face after $72 \mathrm{~m}$ of cryogenic cutting with $p_{w}=7 \mathrm{bar}$ and subcooler usage 


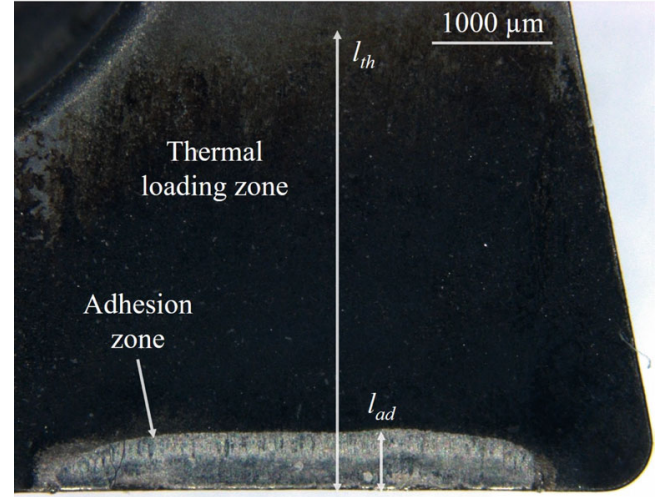

Fig. 16 Tool rake face after $72 \mathrm{~m}$ of dry cutting

- Black edging indicates the area of high thermal loading, where no tool-workpiece-adhesion occurs.

- Faint scratches running from the cutting edge mark occasional friction between tool and chip. These scratches however cannot be identified in dry cutting, as the thermal loading zone covers a large area of the rake face.

The rake faces of the tools were evaluated in terms of adhesion length and thermal loading length, because toolworkpiece-adhesion is a well known wear phenomenon in machining of titanium alloys. The adhesion length can be attributed to the crater width in DIN ISO 3685. Furthermore the flank wear land width VB according to DIN ISO 3685 was evaluated. The results are summarized in Table 5.

Compared with dry cutting, adhesion, thermal loading and VB are remarkably reduced by cryogenic cooling. Within the cryogenic turning experiments, 4 bar working pressure without subcooler clearly leads to the highest wear marks, which is explained by an insufficient cooling. Among the remaining turning experiments the effect of the cooling parameters is less pronounced. Still the length of thermal loading generally reduces with the nitrogen pressure. Regarding cryogenic turning with and without subcooler separately, this is in accordance with the maximum temperatures in Fig. 13. Comparing cutting with and without subcooler, it must be noticed that the unstable nitrogen supply can cause temperature peaks, as shown in Fig. 11. Therefore, the evolution of the mean tool temperature with the working pressure may differ from the maximum temperatures.

While the adhesion length is rarely analyzed in practical applications, a flank wear width of $\mathrm{VB}=300 \mu \mathrm{m}$ is a often taken as an indicator for the end of tool life [5]. Here it must be mentioned that orthogonal turning is an analogy process which is not used in industry. But it can be assumed that the flank wear in dry cutting of $280 \mu \mathrm{m}$ represents a critical value, when the referring passive force increase is considered, which is indicated in Fig. 9. Apart from the cryogenic turning experiment without subcooler at 4 bar working pressure, the flank wear widths in cryogenic cutting are on a common level, i.e. between 180 and 200 $\mu \mathrm{m}$. Besides the before mentioned positive effects of the process cooling on the tool life, negative effect can also be imagined. The tungsten carbides of the uncoated tool are bonded by a cobalt matrix, which is responsible for the toughness of the insert. A strong cooling of the insert could lead to a toughness reduction, resulting in less resistance to cracks and carbide breakouts. To investigate this issue, tool life experiments are planned for longitudinal turning, which is in contrast to orthogonal turning an industrially relevant machining process.

\section{Conclusion}

In this paper, tool internal rake face nitrogen cooling of orthogonal turning of Ti-6Al-4V was investigated. The main findings are briefly summarized:

- Rising the nitrogen working pressure increases the mass flow rate of the cooling system. Nitrogen evaporation in the supply channel causes fluid drag, e.g. a proper heat insulation and initial cooling of the supply system increase the mass flow rate as well.

- Subcooler usage decreases the nitrogen evaporation rate and accelerates the initial cooling of the supply system. Therefore, increased mass flow rates result at the same working pressures, compared to systems without subcooling.

- A gaseous phase fraction in the nitrogen stream leads to temperature peaks in the cooled tool, also during cutting experiments. These peaks are prevented by subcooling.

Table 5 Evaluation of adhesion length $l_{\text {ad }}$, thermal loading length $l_{\text {th }}$ and the flank wear width VB

\begin{tabular}{llllllllll}
\hline $\begin{array}{l}p_{w} \text { in bar } \\
\text { subcooler }\end{array}$ & Dry & 4 & 5 & 6 & 7 & 4 & 5 & $\begin{array}{l}7 \\
\text { Yes }\end{array}$ & Yes \\
\hline$l_{\text {th }}$ in $\mathrm{mm}$ & 3.76 & 1.20 & 1.05 & 0.92 & 1.02 & 1.15 & 1.08 & 1.02 \\
$l_{\text {ad }}$ in $\mu \mathrm{m}$ & 528 & 389 & 357 & 341 & 336 & 359 & 329 & 357 & 333 \\
VB in $\mu \mathrm{m}$ & 281 & 236 & 195 & 199 & 193 & 202 & 180 & 193 \\
\hline
\end{tabular}


Without subcooling, low working pressures lead to continous nitrogen evaporation and an insufficient cooling of the tool system. In this case the subcooler has a strong effect on the process temperatures. Without subcooling, the tested working pressures between 5 and 7 bar were not suited to adjust the mean maximum temperature in a reliable manner. However with subcooling, a clear trend in the cutting temperatures is seen between 4 and 7 bar. Consequently a convenient adjustment of the tool temperature by the working pressure is only possible when using a subcooler. This technique could be used to easily adjust the cooling effect and the $\mathrm{LN}_{2}$ consumption to different machining parameters or workpiece materials.

- Compared to dry cutting, the internal rake face application of nitrogen into the tool-chip-contactzone effectively reduces tool-chip-contact-length, the thermal loading zone and the flank wear width in orthogonal turning. The precondition is that a sufficient process cooling is ensured by the working pressure or subcooler usage. For the given experimental setup, the minimum channel-to-edge-distance of $0.8 \mathrm{~mm}$ and the channel diameter of $1.5 \mathrm{~mm}$ were well suited in terms of cutting edge stability and wear reduction. The detailed dependencies between the tool wear and the working pressure as well as subcooler usage will be subjected to future investigations.

Funding Open Access funding enabled and organized by Projekt DEAL. This work was funded by the DFG within the projects SCHU 1010/51-1 and MA 1205/24-1.

Open Access This article is licensed under a Creative Commons Attribution 4.0 International License, which permits use, sharing, adaptation, distribution and reproduction in any medium or format, as long as you give appropriate credit to the original author(s) and the source, provide a link to the Creative Commons licence, and indicate if changes were made. The images or other third party material in this article are included in the article's Creative Commons licence, unless indicated otherwise in a credit line to the material. If material is not included in the article's Creative Commons licence and your intended use is not permitted by statutory regulation or exceeds the permitted use, you will need to obtain permission directly from the copyright holder. To view a copy of this licence, visit http:// creativecommonshorg/licenses/by/4.0/.

\section{References}

1. Tai BL, Dasch JM, Shih AJ (2011) Evaluation and comparison of lubricant properties in minimum quantity lubrication machining. Mach Sci Technol 15:376-391

2. Busch K, Hochmuth C, Pause B, Stoll A, Wertheim R (2016) Investigation of cooling and lubrication strategies for machining high-temperature alloys. Procedia CIRP 41:835-840

3. Min W, Min W, Youzhen Z (1988) Diffusion wear in milling titanium alloys. Mater Sci Technol 4(6):548-553
4. Yang X, Richard Liu C (1999) Machining titanium and its alloys. Mach Sci Technol 3(1):107-139

5. Arrazola PJ, Garay A, Iriarte LM, Armendia M, Marya S, Le Maître F (2009) Machinability of titanium alloys (Ti6Al4V and Ti555.3). J Mater Process Technol 209(5):2223-2230

6. Hong SY, Ding Y (2001) Cooling approaches and cutting temperatures in cryogenic machining of Ti-6Al-4V. Int J Mach Tools Manuf 41:1417-1437

7. Bermingham MJ, Kirsch J, Sun S, Palanisamy S, Dargusch MS (2011) New observations on tool life, cutting forces and chip morphology in cryogenic machining Ti-6Al-4V. Int J Mach Tools Manuf 51:500-511

8. Klocke F, Settineri L, Lung D, Claudio Priarone P, Arft M (2013) High performance cutting of gamma titanium aluminides: influence of lubricoolant strategy on tool wear and surface integrity. Wear 302:1136-1144

9. Bordin A, Bruschi S, Ghiotti A, Bariani PF (2015) Analysis of tool wear in cryogenic machining of additive manufactured Ti6Al4V alloy. Wear 328-329:89-99

10. Aramcharoen A (2016) Influence of cryogenic cooling on tool wear and chip formation in turning of titanium alloy. Procedia CIRP 46:83-86

11. Trabelsi S, Morel A, Germain G, Bouaziz Z (2017) Tool wear and cutting forces under cryogenic machining of titanium alloy (Ti17). Int J Adv Manuf Tech 91:1493-1505

12. Dix M, Wertheim R, Schmidt G, Hochmuth C (2014) Modeling of drilling assisted by cryogenic cooling for higher efficiency. CIRP Ann Manuf Technol 63:73-76

13. Pusavec F, Lu T, Courbon C, Rech J, Aljancic U, Kopac J, Jawahir IS (2016) Analysis of the influence of nitrogen phase and surface heat transfer coefficient on cryogenic machining performance. $\mathrm{J}$ Mater Process Technol 233:19-28

14. Jawahir IS, Attia H, Biermann D, Duflou J, Klocke F, Meyer D, Newman ST, Pusavec F, Putz M, Rech J, Schulze V, Umbrello D (2016) Cryogenic manufacturing processes. CIRP Ann Manuf Technol 65:713-736

15. Vader DT, Chrysler GM, Chu RC, Simons RE (1995) Experimental investigation of subcooled liquid nitrogen impingement cooling of a silicon chip. IEEE Trans Compon Packaging Manuf Technol Part A 18(4):788-794

16. Lequien P, Poulachon G, Outeiro JC, Rech J (2018) Hybrid experimental/modelling methodology for identifying the convective heat transfer coefficient in cryogenic assisted machining. Appl Therm Eng 128:500-507

17. Lequien P, Poulachon G, Outeiro JC (2018) Thermomechanical analysis induced by interrupted cutting of Ti6Al4V under several cooling strategies. CIRP Ann Manuf Technol 67:91-94

18. Stampfer B, Golda P, Zanger F, Schießl R, Maas U, Schulze V (2019) Thermomechanically coupled numerical simulation of cryogenic orthogonal cutting. Procedia CIRP 82:438-443

19. Jacbosen RT, Stewart RB (1973) Thermodynamic properties of nitrogen including liquid and vapor phases from $63 \mathrm{~K}$ to 2000 $\mathrm{K}$ with pressures up to 10000 bars. Department of Mechanical Engineering, University of Idaho

20. Golda P, Schießl R, Maas U (2019) Heat transfer simulation of a cryogenic cooling stream in machining operation. Int J Heat Mass Transfer 144:118616

21. Pušavec F, Grguraš D, Koch M, Krajnik P (2019) Cooling capability of liquid nitrogen and carbon dioxide in cryogenic milling. CIRP Ann 68(1):73-76

22. Hong SY, Ding Y, Jeong J (2002) Experimental evaluation of friction coefficient and liquid nitrogen lubrication effect in cryogenic machining. Mach Sci Technol 6:235-250

Publisher's note Springer Nature remains neutral with regard to jurisdictional claims in published maps and institutional affiliations. 